\title{
Inhibitory Effect of 1,5-Dimethyl Citrate from Sea Buckthorn (Hippophae rhamnoides) on Lipopolysaccharide-Induced Inflammatory Response in RAW 264.7 Mouse Macrophages
}

\author{
Su Cheol Baek ${ }^{1,+}$, Dahae Lee ${ }^{2,+}$, Mun Seok Jo ${ }^{1}$, Kwang Ho Lee ${ }^{1}$, Yong Hoon Lee ${ }^{1}$, \\ Ki Sung Kang ${ }^{2}$, Noriko Yamabe ${ }^{2, *}$ and Ki Hyun Kim ${ }^{1, *(D)}$ \\ 1 School of Pharmacy, Sungkyunkwan University, Suwon 16419, Korea; schii513@daum.net (S.C.B.); \\ anstjr920827@gmail.com (M.S.J.); sholaly@naver.com (K.H.L.); yhl2090@naver.com (Y.H.L.) \\ 2 College of Korean Medicine, Gachon University, Seongnam 13120, Korea; pjsldh@naver.com (D.L.); \\ kkang@gachon.ac.kr (K.S.K.) \\ * Correspondence: norikoy@gachon.ac.kr (N.Y.); khkim83@skku.edu (K.H.K.); Tel.: +82-31-290-7700 (K.H.K.) \\ + These authors contributed equally to this work.
}

Received: 29 November 2019; Accepted: 7 February 2020; Published: 2 March 2020

Abstract: Hippophae rhamnoides L. (Elaeagnaceae; commonly known as "sea buckthorn" and "vitamin tree"), is a spiny deciduous shrub whose fruit is used in foods and traditional medicines. The $H$. rhamnoides fruit (berry) is rich in vitamin $\mathrm{C}$, with a level exceeding that found in lemons and oranges. $H$. rhamnoides berries are usually washed and pressed to create pomace and juice. Today, the powder of the aqueous extract of $H$. rhamnoides berries are sold as a functional food in many countries. As part of our ongoing effort to identify bioactive constituents from natural resources, we aimed to isolate and identify those from the fruits of $H$. rhamnoides. Phytochemical analysis of the extract of H. rhamnoides fruits led to the isolation and identification of six compounds, namely, a citric acid derivative (1), a phenolic (2), flavonoids ( 3 and 4 ), and megastigmane compounds (5 and $\mathbf{6}$ ). Treatment with compounds 1-6 did not have any impact on the cell viability of RAW 264.7 mouse macrophages. However, pretreatment with these compounds suppressed lipopolysaccharide (LPS)-induced NO production in RAW 264.7 mouse macrophages in a concentration-dependent manner. Among the isolated compounds, compound 1 was identified as the most active, with an $\mathrm{IC}_{50}$ of $39.76 \pm 0.16 \mu \mathrm{M}$. This value was comparable to that of the $N^{\mathrm{G}}$-methyl-L-arginine acetate salt, a nitric oxide synthase inhibitor with an $\mathrm{IC}_{50}$ of $28.48 \pm 0.05 \mu \mathrm{M}$. Western blot analysis demonstrated that compound 1 inhibited the LPS-induced expression of IKK $\alpha / \beta$ (I $\kappa B$ kinase alpha/beta), I- $\kappa \mathrm{B} \alpha$ (inhibitor of kappa $\mathrm{B}$ alpha), nuclear factor kappa-B (NF-kB) p65, iNOS (inducible nitric oxide synthase), and COX-2 (cyclooxygenase-2) in RAW 264.7 cells. Furthermore, LPS-stimulated cytokine production was detected using a sandwich enzyme-linked immunosorbent assay. Compound 1 decreased interleukin 6 (IL-6) and tumor necrosis factor alpha (TNF- $\alpha$ ) production in LPS-stimulated RAW 264.7 cells. In summary, the mechanism of action of $\mathbf{1}$ included the suppression of LPS-induced NO production in RAW 264.7 cells by inhibiting IKK $\alpha / \beta, \mathrm{I}-\kappa \mathrm{B} \alpha, \mathrm{NF}-\mathrm{\kappa B}$ p65, iNOS, and COX-2, and the activities of IL-6 and TNF- $\alpha$.

Keywords: Hippophae rhamnoides; sea buckthorn; citric acid derivative; NO production; RAW 264.7 cells

\section{Introduction}

Hippophae rhamnoides L. (commonly known as 'Sea buckthorn' and 'Vitamin tree') is a spiny deciduous shrub belonging to the Elaeagnaceae family. The fruit of $H$. rhamnoides is rich in vitamin $\mathrm{C}$ 
and its levels exceed those found in lemons and oranges [1]. The berries of H. rhamnoides are usually washed and pressed to create pomace and juice. In many countries, the powder from the aqueous extract of $H$. rhamnoides berries are consumed as a functional food. The berry of H. rhamnoides is used as food and medicine in Asia, specifically in Tibetan and Mongolian medicines, owing to its antibechic, antiviral, and antioxidant activities [2,3]. Moreover, the berries are used to make fruit sauce, fruit powder, and fruit wine [4]. Previous biological studies of the H. rhamnoides extracts revealed their anti-platelet effect through the inhibition of thrombin-activated platelets to collagen or fibrinogen [5]. The phenolic and nonpolar lipid fractions of the H. rhamnoides extracts attenuate the essential pathogenic properties of Staphylococcus aureus and Candida albicans, and the mechanism of the effect was found to be due to a decrease in adhesion and biofilm formation on inert surfaces [6]. Previously, a study found that the pomace of H. rhamnoides exerts antioxidant activity [7]. Therefore, because of the health benefits of $H$. rhamnoides, its phytochemical constituents were investigated, and further demonstrated to include flavonoids, alkaloids, protocatechuic acid, and triterpenoid glycosides [8-10]. Among them, isorhamnetin 3,5,7,4-tetrahydroxy-3-methoxyflavon, isorhamnetin 3-O- $\beta$-D-glucopyranosil-(6-1)-O- $\alpha$-1-rhamnopyranoside, and protocatechuic acid were found to have a regulatory effect on hepatic stellate cell activation and liver fibrogenesis by affecting changes in DNA expression and cell cycle arrest, and decreasing the release of cytokines [11].

As natural products are rich sources of anti-inflammatory agents [12,13], evaluating their anti-inflammatory effects using laboratory models is important for drug discovery. Inflammation is a critical immune response to different factors, such as bacteria, chemicals, and viruses, that result in inflammation-mediated diseases, including inflammatory bowel disease, cancer, atherosclerosis, diabetes, and asthma [14]. Discovering the bioactive constituents present in natural products can thus serve as an approach to prevent and treat inflammation-mediated diseases. Numerous laboratory models are available for screening and assessing the anti-inflammatory agents derived from natural products [12]. Through in vitro and in vivo laboratory models, macrophages have been identified as the principal cell type in the innate immune system [15]. Lipopolysaccharides (LPS), which are known as an endotoxin of Gram-negative bacteria, have been widely used to study the inflammatory response in macrophages. This is because activated macrophages release $\mathrm{NO}$ as an indicator of pro-inflammatory reactions in response to LPS $[16,17]$. During the above process, the nuclear factor kappa-B (NF- $\mathrm{kB}$ ) proteins translocate to the nucleus from the cytoplasm and induce the transcription of pro-inflammatory mediators and cytokines [18]. Thus, the ability of NF- $\mathrm{KB}$ to modulate the expression of cyclooxygenase-2 (COX-2), inducible nitric oxide synthase (iNOS), interleukin 6 (IL-6), and tumor necrosis factor alpha $(\mathrm{TNF} \alpha)$ allows us to assess the anti-inflammatory effect of natural products against inflammatory response.

As part of our ongoing research to discover the bioactive constituents in natural sources [19-22], here we elucidated the bioactive compounds in the fruits of $H$. rhamnoides. Through a phytochemical analysis of the extract from $H$. rhamnoides fruits, we isolated and identified six compounds, including a citric acid derivative (1), a phenolic (2), two flavonoids (3 and 4), and two megastigmane compounds (5 and 6), through a comparison of their nuclear magnetic resonance (NMR) spectroscopic data to their reported values and the results of LC/MS analysis. In the present study, we sought to report the anti-inflammatory effect of the constituents of H. rhamnoides and elucidate the underlying molecular mechanism whereby the active compound 1 modulates the NF- $\mathrm{KB}$ p 65 signaling pathway, which affects the LPS-induced inflammatory responses in RAW 264.7 mouse macrophages.

\section{Materials and Methods}

\subsection{General Experimental Procedures}

Ultraviolet (UV) spectra were acquired on an Agilent 8453 UV-visible spectrophotometer (Agilent Technologies, Santa Clara, CA, USA). Experimental electronic circular dichroism (ECD) spectra in methanol $(\mathrm{MeOH})$ were acquired in a quartz cuvette of $1 \mathrm{~mm}$ optical path length on a JASCO J-1500 
spectropolarimeter (Tokyo, Japan). Nuclear magnetic resonance (NMR) spectra were recorded on a Bruker AVANCE III HD 850 NMR spectrometer with a $5 \mathrm{~mm}$ TCI CryoProbe operated at $850 \mathrm{MHz}$ $\left({ }^{1} \mathrm{H}\right)$ and $212.5 \mathrm{MHz}\left({ }^{13} \mathrm{C}\right)$ (Bruker, Karlsruhe, Germany). For the ${ }^{1} \mathrm{H}$ and ${ }^{13} \mathrm{C}$ NMR analyses, chemical shifts are expressed in ppm ( $\delta$ ). Medium-pressure liquid chromatography (MPLC) was conducted using Smart Flash AKROS (Yamazen, Osaka, Japan). Semi-preparative HPLC was carried out using a Shimadzu Prominence HPLC System with SPD-20A/20AV Series Prominence HPLC UV-VIS detectors (Shimadzu, Tokyo, Japan) and a Phenomenex Luna C18 column $(250 \times 10 \mathrm{~mm}, 5 \mu \mathrm{m}$; flow rate: 2 $\mathrm{mL} / \mathrm{min}$; Phenomenex, Torrance, CA, USA). LC/MS analysis was performed on an Agilent 1200 Series HPLC system equipped with a diode array detector, a 6130 Series ESI mass spectrometer, and an analytical Kinetex C18 $100 \AA ̊$ column $(100 \times 2.1 \mathrm{~mm}, 5 \mu \mathrm{m}$; flow rate: $0.3 \mathrm{~mL} / \mathrm{min}$; Phenomenex). Silica gel 60 (230-400 mesh; Merck, Darmstadt, Germany) and RP-C 18 silica gel (Merck, 230-400 mesh) were used for column chromatography. Sephadex LH-20 (Pharmacia, Uppsala, Sweden) was employed as the packing material for molecular sieve column chromatography. Merck precoated silica gel $\mathrm{F}_{254}$ plates and RP- $\mathrm{C}_{18} \mathrm{~F}_{254 \mathrm{~s}}$ plates were used for thin-layer chromatography (TLC). Following TLC, spots were detected under UV light or by heating when sprayed with anisaldehyde-sulfuric acid.

\subsection{Plant Material}

The powder of H. rhamnoides fruits was purchased in October of 2018 from Korea Beauty and Healthcare Co., Ltd. Thereafter, the material was identified by one of the authors (K.H.K). A voucher specimen of the material (VT-2018) was deposited in the herbarium at the School of Pharmacy, Sungkyunkwan University, Suwon, Republic of Korea.

\subsection{Extraction and Isolation}

The aqueous extract powder $(270 \mathrm{~g})$ of $\mathrm{H}$. rhamnoides fruits was suspended in distilled water (700 mL) prior to solvent partitioning with $n$-hexane, dichloromethane $\left(\mathrm{CH}_{2} \mathrm{Cl}_{2}\right)$, ethyl acetate (EtOAc), and $n$-butanol $(\mathrm{BuOH})$ to obtain four partitioned layers: hexane-soluble $(2.7 \mathrm{~g})$, methylene chloride (MC)-soluble (3.4 g), ethyl acetate (EA)-soluble (7.8 g), and BuOH-soluble (25.2 g) layers. The MC-soluble layer (3.4 g) was subjected to silica gel column chromatography (100 g, eluted with MC/MeOH (100:1 $\rightarrow$ 1:1); gradient solvent system, washed with $100 \% \mathrm{MeOH}$ ) to yield seven fractions (C1-C7). Fraction C4 $(55.7 \mathrm{mg})$ was separated via MPLC on a Yamazen UNIVERSAL Premium silica column with MC/MeOH $(100: 0 \rightarrow 1: 1)$ to yield four subfractions (C41-C44). Subfraction C41 (46.4 mg) was separated on a Sep-pak $\mathrm{C} 18$ cartridge using a solvent system consisting of $90 \% \mathrm{MeOH}$ to yield three subfractions (C411-C413). Subfraction C411 (17.4 mg) was subsequently purified by semi-preparative HPLC (35\% $\mathrm{MeOH})$ on a Phenomenex Luna C18 column to yield compound 5 ( $t_{\mathrm{R}} 36.5 \mathrm{~min}, 0.5 \mathrm{mg}$ ). Fraction C5 (74.5 mg) was purified by semi-preparative HPLC ( $39 \% \mathrm{MeOH})$ on a Phenomenex Luna C18 column to yield compound $6\left(t_{\mathrm{R}} 23.0 \mathrm{~min}, 1.5 \mathrm{mg}\right)$. The EA-soluble layer $(7.8 \mathrm{~g})$ was chromatographed by silica gel column chromatography (200 g, eluted with $\mathrm{MC} / \mathrm{MeOH}(20: 1 \rightarrow 1: 1)$; gradient solvent system, washed with $100 \% \mathrm{MeOH}$ ) to yield seven fractions (E1-E7). Fraction E3 (751.1 mg) was separated on a Sephadex LH-20 column using a solvent system of $100 \% \mathrm{MeOH}$ to yield five subfractions (E31-E35). Subfraction E35 (15.7 mg) was purified by semi-preparative HPLC (19\% acetonitrile) on a Phenomenex Luna C18 column to yield compound $2\left(t_{\mathrm{R}} 34.8 \mathrm{~min}, 1.0 \mathrm{mg}\right)$. Fraction E4 (1.37 g) was separated on a Sephadex LH-20 column using a solvent system of $100 \% \mathrm{MeOH}$ to yield three subfractions (E41-E43). Subfraction E42 (1.24 g) was subjected to silica gel column chromatography (100 g, eluted with $\mathrm{MC} / \mathrm{MeOH}(20: 1 \rightarrow 1: 1)$; gradient solvent system, washed with $80 \% \mathrm{MeOH})$ to yield six subfractions (E421-E426). Subfraction E422 (391.3 mg) was separated by reversed-phase preparative HPLC on an Agilent Eclipse C18 column with a gradient solvent system of $\mathrm{MeOH} / \mathrm{H}_{2} \mathrm{O}(10 \%-70 \%-100 \%)$ to yield five subfractions (E4221-E4225). Subfraction E4223 was purified by semi-preparative HPLC (20\% $\mathrm{MeOH}$ ) on a Phenomenex Luna C18 column to yield subfraction E42236. Subfraction E42236 (23.0 mg) was re-purified by semi-preparative HPLC $(10 \% \mathrm{MeOH})$ on a Phenomenex Luna C18 column to yield compound $1\left(t_{\mathrm{R}} 37.5 \mathrm{~min}, 6.0 \mathrm{mg}\right)$. Subfraction E43 (55.3 mg) was separated by semi-preparative HPLC 
$(15 \% \mathrm{MeOH})$ to yield six subfractions (E431-E436). Subfraction E436 was purified by semi-preparative HPLC ( $38 \% \mathrm{MeOH})$ on a Phenomenex Luna C18 column to yield compounds 3 ( $t_{\mathrm{R}} 56.6 \mathrm{~min}, 0.6 \mathrm{mg}$ ) and $4\left(t_{\mathrm{R}} 58.5 \mathrm{~min}, 0.9 \mathrm{mg}\right)$.

\subsection{RAW 264.7 Cell Culture}

The mouse macrophage cell line, RAW 264.7 (American Type Culture Collection, Rockville, MD, USA), was cultured in Dulbecco's modified Eagle's medium (Cellgro, VA, USA) containing 10\% fetal bovine serum (FBS) and $1 \%$ penicillin/streptomycin (Invitrogen Co., NY, USA) at $37^{\circ} \mathrm{C}$ in an atmosphere of $5 \% \mathrm{CO}_{2}$.

\subsection{Measurement of RAW 264.7 Cell Viability}

RAW 264.7 cell viability was determined using an Ez-Cytox cell viability detection kit (Daeil Lab Service Co., Seoul, Korea). Cells were plated in 96-well plates at a density of $3 \times 10^{4}$ cells/well and incubated for $24 \mathrm{~h}$ at $37^{\circ} \mathrm{C}$. Cells were exposed to compounds at concentrations of 5, 10, 25, 50, and $100 \mu \mathrm{M}$ for $1 \mathrm{~h}$ at $37^{\circ} \mathrm{C}$. Thereafter, they were incubated with vehicle (medium containing $0.5 \%$ DMSO) for $24 \mathrm{~h}$ at $37^{\circ} \mathrm{C}$. After that, they were incubated with the Ez-Cytox solution for $40 \mathrm{~min}$ at $37^{\circ} \mathrm{C}$. Absorbance was determined at $450 \mathrm{~nm}$ using a PowerWave XS microplate reader (Bio-Tek Instruments, Winooski, VT, USA).

\subsection{Measurement of NO Production in RAW 264.7 Cells}

RAW 264.7 cells were plated in 96-well plates at a density of $3 \times 10^{4}$ cells/well for a $24 \mathrm{~h}$ incubation at $37^{\circ} \mathrm{C}$. Cells were either exposed to the compounds or $N^{\mathrm{G}}$-methyl-L-arginine acetate salt (L-NMMA) at concentrations of $5,10,25,50$, and $100 \mu \mathrm{M}$ for $1 \mathrm{~h}$ at $37^{\circ} \mathrm{C}$. Thereafter, they were incubated with $1 \mu \mathrm{g} / \mathrm{mL} \mathrm{LPS} \mathrm{for} 24 \mathrm{~h}$ at $37^{\circ} \mathrm{C}$. The supernatant was mixed with an equal volume of Griess reagent containing $0.2 \%$ naphthylethylenediamine dihydrochloride (Sigma-Aldrich, St. Louis, MO, USA) and $2 \%$ sulfanilamide (Sigma-Aldrich) in 5\% phosphoric acid (Sigma-Aldrich) and incubated for $40 \mathrm{~min}$ at $37^{\circ} \mathrm{C}$. The absorbance was then measured at $540 \mathrm{~nm}$ using a PowerWave XS microplate reader.

\subsection{Western Blot Analysis}

RAW 264.7 cells were plated in 6-well plates at a density of $2 \times 10^{5}$ cells/well and incubated for $24 \mathrm{~h}$ at $37^{\circ} \mathrm{C}$. Cells were exposed to 50 and $100 \mu \mathrm{M}$ of compound $1 \mathrm{for} 1 \mathrm{~h}$ at $37^{\circ} \mathrm{C}$ prior to incubation with $1 \mu \mathrm{g} / \mathrm{mL}$ LPS for $24 \mathrm{~h}$ at $37^{\circ} \mathrm{C}$. Whole-cell extracts were then prepared using radio immunoprecipitation assay buffer (RIPA buffer; Cell Signaling Technology, Inc., Beverly, MA, USA) containing $1 \times$ ethylenediaminetetraacetic acid (EDTA)-free protease inhibitor cocktail and 1 $\mathrm{mM}$ phenylmethylsulfonyl fluoride (PMSF), according to the manufacturer's instructions. Protein concentration of the whole-cell extracts was determined with a bicinchoninic acid (BCA) protein assay kit. Proteins $(20 \mu \mathrm{g} / \mathrm{lane})$ were subjected to $10 \%$ sodium dodecyl sulfate polyacrylamide gel electrophoresis (SDS-PAGE) for $90 \mathrm{~min}$ at $110 \mathrm{~V}$, transferred to a polyvinylidene fluoride (PVDF) membrane, and analyzed with epitope-specific primary antibodies to phospho-IкB kinase (IKK) $\alpha / \beta$,

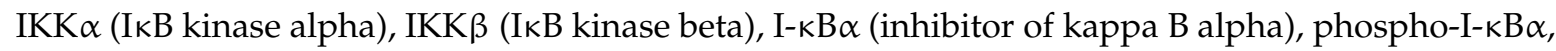
phospho-NF- $k B$ p65, NF- $\kappa B$ p65, and iNOS, followed by cyclooxygenase-2 (COX-2), glyceraldehyde 3-phosphate dehydrogenase (GAPDH), and horseradish peroxidase (HRP)-conjugated anti-rabbit antibodies (Cell Signaling, MA, USA). The bound antibodies were then visualized with ECL Advance Western Blotting Detection Reagents (GE Healthcare, Cambridge, United Kingdom) and a FUSION Solo Chemiluminescence System (PEQLAB Biotechnologie GmbH, Erlangen, Germany). The optical densities of the immunoreactive bands were obtained using ImageJ software (Version 1.51J; National Institutes of Health, Bethesda, MD, USA) and normalized with those of the control. Data represented by fold-increases were compared to the control. The GAPDH was used for loading control. 


\subsection{Determination of IL-6 and TNF- $\alpha$ Production}

RAW 264.7 cells were plated in 24-well plates at a density of $4 \times 10^{5}$ cells/well and incubated for $24 \mathrm{~h}$ at $37^{\circ} \mathrm{C}$. Cells were then exposed to compound 1 at concentrations of 50 and $100 \mu \mathrm{M}$ for $1 \mathrm{~h}$ at $37^{\circ} \mathrm{C}$ prior to incubation with $1 \mu \mathrm{g} / \mathrm{mL}$ LPS for $24 \mathrm{~h}$ at $37^{\circ} \mathrm{C}$. To determine IL- 6 and tumor necrosis factor alpha (TNF- $\alpha$ ) production, the supernatant was obtained and assayed to quantify the levels of these cytokines using an IL-6 sandwich enzyme-linked immunosorbent assay (ELISA) kit (BD Biosciences, CA, USA) and a TNF- $\alpha$ ELISA kit (eBiosciences, CA, USA), according to the manufacturer's instructions.

\subsection{Statistical Analysis}

At least three independent determinations were carried out for each experiment. All data are presented as average value and standard deviation (SD). Statistical significance was determined using one-way analysis of variance (ANOVA), and multiple comparisons were carried out with a Bonferroni correction. A $p$-value less than 0.05 indicated statistical significance. All statistical analyses were performed using SPSS Statistics version 19.0 (SPSS Inc., Chicago, IL, USA).

\section{Results and Discussion}

\subsection{Isolation and Identification of Compounds}

The aqueous extract powder of $H$. rhamnoides fruits was first suspended in distilled water, and then subjected to solvent-partitioning with $n$-hexane, $\mathrm{CH}_{2} \mathrm{Cl}_{2}$, ethyl acetate, and $n$ - $\mathrm{BuOH}$ to obtain each solvent fraction. Phytochemical analysis of the $\mathrm{CH}_{2} \mathrm{Cl}_{2}$-soluble and ethyl acetate-soluble fractions was performed using repeated column chromatography, HPLC, and LC/MS. Through chemical analysis, six compounds (1-6), including a citric acid derivative (1), a phenolic (2), two flavonoids (3 and 4), and two megastigmane compounds (5 and 6) (Figure 1) were isolated; compounds 1-4 were isolated from the ethyl acetate-soluble fraction whereas compounds 5-6 were isolated from the $\mathrm{CH}_{2} \mathrm{Cl}_{2}$-soluble fraction. By comparing the NMR spectroscopic data of the compounds to their reported values and the LC/MS results, we identified that the compounds were 1,5-dimethyl citrate (1) [23], 5-methoxysalicylic acid (2) [24], syringetin-3-O-glucoside (3) [25], isorhamnetin-3-O-glucoside (4) [9], (+)-dehydrovomifoliol (5) [26], and (+)-vomifoliol (6) [27]. In particular, the absolute configurations for compounds 5 and 6 were established by their positive specific rotation values and comparison of their NMR spectroscopic data with those reported earlier and electronic circular dichroism (ECD) data (Figure S1) [26,27].<smiles>COC(=O)CC(O)(CC(=O)OC)C(=O)O</smiles>

Figure 1. Chemical structures of compounds 1-6. 1,5-Dimethyl citrate (1), 5-methoxysalicylic acid (2), syringetin-3-O-glucoside (3), isorhmanetin-3-O-glucoside (4), (+)-dehydrovomifoliol (5), and (+)-vomifoliol (6). 


\subsection{Effects of Compounds 1-6 on Cell Viability}

Prior to evaluating the effect of the compounds on NO production, we determined their cytotoxicity in RAW 264.7 mouse macrophages using an Ez-Cytox cell viability assay. As a result, the compounds were considered to be cytotoxic when the cell viability in the compound-treated group was less than $80 \%$ of that in the untreated group. At all tested concentrations, all concentrations of compounds 1-6 had no cytotoxicity on RAW 264.7 mouse macrophages (Figure 2). Therefore, all concentrations were employed for the subsequent experiments.

(A)

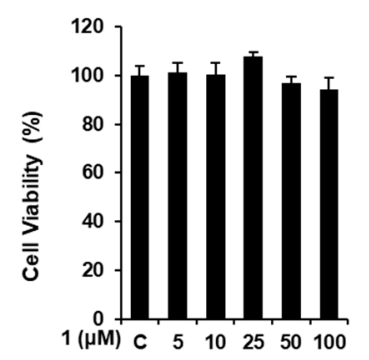

(D)

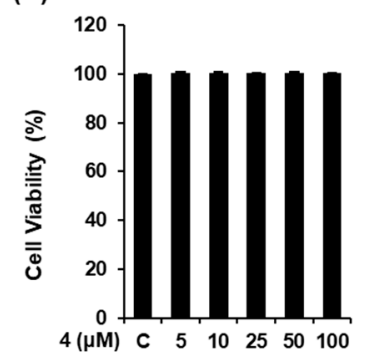

(B)

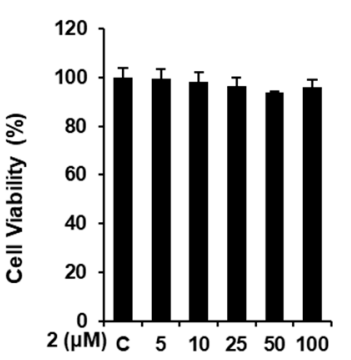

(E)

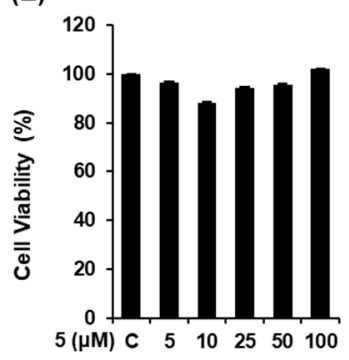

(C)

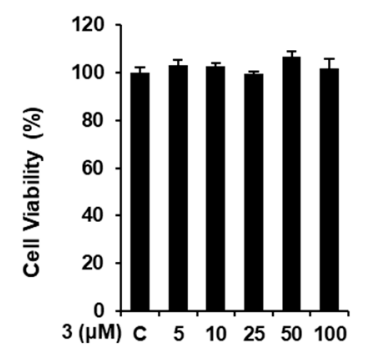

(F)

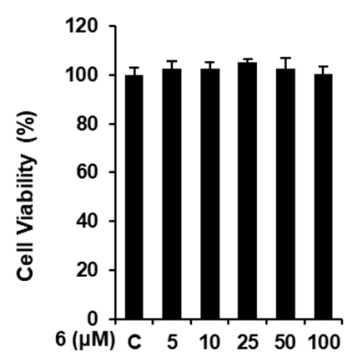

Figure 2. Effect of compounds 1-6 on the viability of RAW 264.7 mouse macrophages. (A-F) The viability of RAW 264.7 cells incubated with compounds 1-6 for $24 \mathrm{~h}$ was measured using an Ez-Cytox cell viability assay (mean $\pm \mathrm{SD},{ }^{*} p<0.05$ compared to the lipopolysaccharide (LPS)-treated group). C: control group treated with 0.5\% DMSO. 1,5-dimethyl citrate (1), 5-methoxysalicylic acid (2), syringetin-3-O-glucoside (3), isorhmanetin-3-O-glucoside (4), (+)-dehydrovomifoliol (5), and (+)-vomifoliol (6).

\subsection{Effects of Compounds 1-6 on NO Production}

The production of NO in LPS-induced RAW 264.7 macrophages has been used as an indicator to evaluate the anti-inflammatory effects of natural products. In the present study, we evaluated the effect of compounds 1-6 on LPS-induced NO production in RAW 264.7 cells. In this assay, L-NMMA, a nitric oxide synthase inhibitor, was used as the positive control. As shown in Figure 3, exposure to $1 \mu \mathrm{g} / \mathrm{mL}$ LPS induced a significant increase in NO production; however, this was suppressed by pretreatment with compounds 1-6, which had 50\% inhibitory concentration $\left(\mathrm{IC}_{50}\right)$ values of $39.76 \pm$ $0.16 \mu \mathrm{M}, 79.39 \pm 0.29 \mu \mathrm{M}, 86.33 \pm 0.54 \mu \mathrm{M}, 87.01 \pm 0.30 \mu \mathrm{M}, 74.71 \pm 0.24 \mu \mathrm{M}$, and $76.12 \pm 0.14 \mu \mathrm{M}$, respectively. Although the $\mathrm{IC}_{50}$ values of most compounds were slightly weaker than that of L-NMMA $\left(\mathrm{IC}_{50}, 28.48 \pm 0.05 \mu \mathrm{M}\right)$, compound 1 displayed a similar $\mathrm{IC}_{50}$ value, which indicates that it might inhibit the increase of LPS-induced NO production in RAW 264.7 macrophages. This finding suggests that the inflammatory responses induced by extracellular stimuli might be suppressed via treatment with compound $\mathbf{1}$. Thus, we proceeded to investigate the potential benefit of compound $\mathbf{1}$ as an NO production inhibitor by carrying out a mechanism study. 
(A)

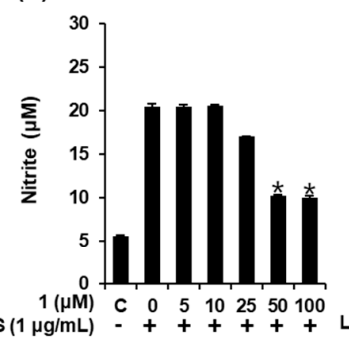

(B)

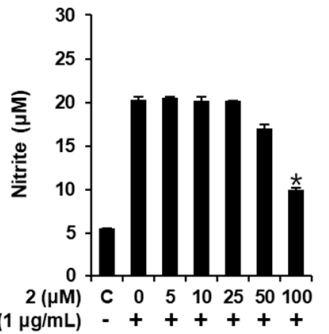

(C)

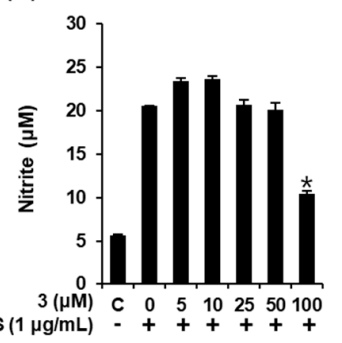

(D)

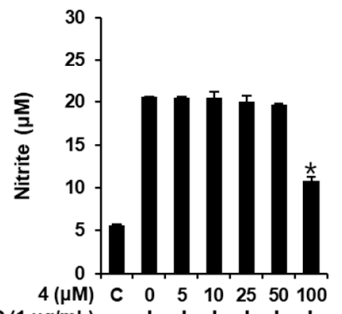

(E)

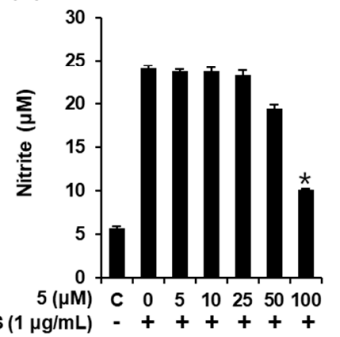

(F)

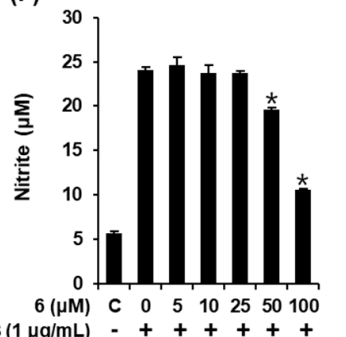

(G)

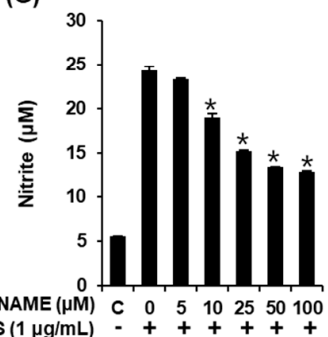

Figure 3. Effect of compounds 1-6 on the LPS-stimulated NO production in RAW 264.7 mouse macrophages. The effect of (A-F) the compounds and $(\mathbf{G}) N^{\mathrm{G}}$-methyl-L-arginine acetate salt (L-NMMA) in LPS-treated RAW 264.7 macrophages was detected using the Griess reagent (mean \pm SD, * $p<$ 0.05 compared to the LPS-treated group). C: control group treated with 0.5\% DMSO. 1,5-Dimethyl citrate (1), 5-methoxysalicylic acid (2), syringetin-3-O-glucoside (3), isorhmanetin-3-O-glucoside (4), (+)-dehydrovomifoliol (5), and (+)-vomifoliol (6).

3.4. Compound 1 Downregulated IKK $\alpha / \beta, I-\kappa B \alpha$, and NF- $\kappa B$ p65 in LPS-Stimulated RAW 264.7 Mouse Macrophages

When macrophages are stimulated with LPS, IKK $\alpha / \beta$ is phosphorylated, thereby leading to the subsequent phosphorylation and degradation of $\operatorname{I} \kappa \mathrm{B} \alpha$, which enables the nuclear translocation of NF- $\kappa B$ [28]. NF- $\kappa B$ p 65 is a core mediator of the innate immune response that leads to the transcription of genes encoding pro-inflammatory mediators, such as iNOS and COX-2, and pro-inflammatory cytokines, such as TNF- $\alpha$ and IL-6 $[29,30]$. Therefore, to confirm whether pre-treatment with compound 1 could suppress LPS-stimulated NO production through the NF- $\mathrm{B}$ p65 signaling pathway, we determined the effect of compound 1 on the expression of IKK $\alpha / \beta, I-\kappa B \alpha$, and NF- $\kappa B$ p65 in LPS-stimulated RAW 264.7 mouse macrophages. As shown in Figure 4, exposure to $1 \mu \mathrm{g} / \mathrm{mL}$ LPS significantly upregulated $\mathrm{IKK} \alpha / \beta, \mathrm{I}-\kappa \mathrm{B} \alpha$, and NF- $\mathrm{B}$ p65; however, pretreatment with 50 and $100 \mu \mathrm{M}$ of compound 1 reversed these changes induced by LPS. Such findings indicate that compound 1 inhibits NO production by inhibiting the phosphorylation of IKK $\alpha / \beta, \mathrm{I}-\kappa \mathrm{B} \alpha$, and NF- $\mathrm{KB}$ p65 in RAW 264.7 macrophages. On the basis of these results, it appears that the action site of compound $\mathbf{1}$ is upstream of the IKK site, thus modulating inflammatory mediator expression. 
(A)

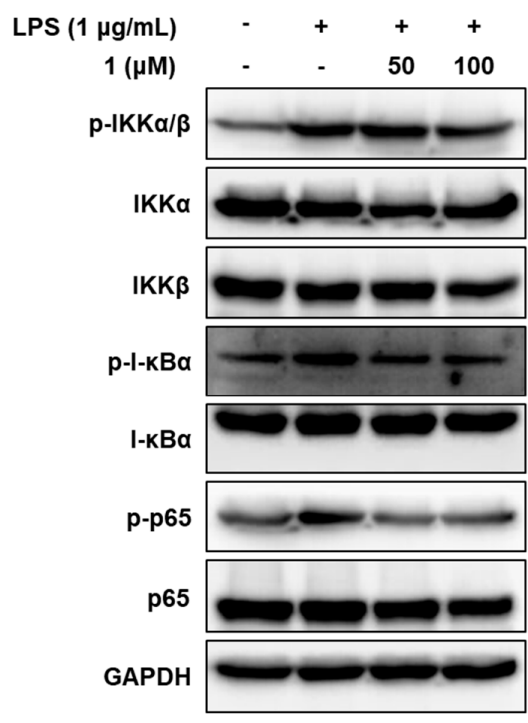

(B)

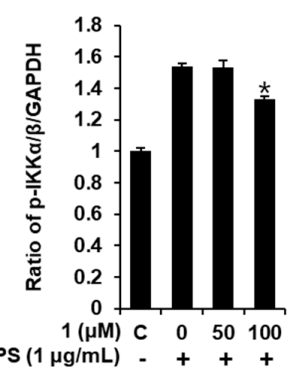

(D)

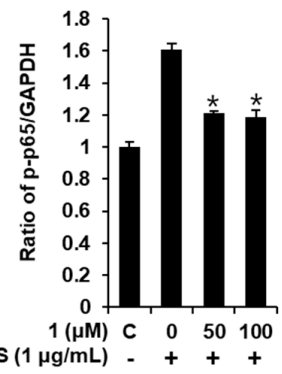

(C)

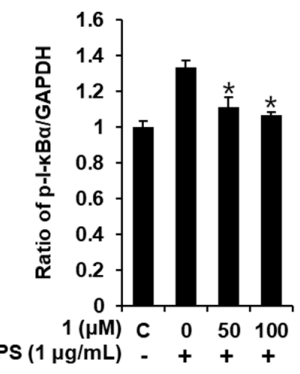

Figure 4. Effects of compound 1 on the LPS-induced expression of the IKK $\alpha / \beta$ (IKB kinase alpha/beta), $\mathrm{I}-\mathrm{kB} \alpha$ (inhibitor of kappa B alpha), and NF-kB p65 proteins in RAW 264.7 mouse macrophages. (A) Representative Western blots of IKK $\alpha / \beta, I-\kappa B \alpha$, NF- $\kappa B$ p65, and glyceraldehyde 3-phosphate dehydrogenase (GAPDH) protein expression. Quantitative graph of $(\mathbf{B}) \mathrm{p}-\mathrm{IKK} \alpha / \beta,(\mathbf{C}) \mathrm{p}-\mathrm{I}-\kappa \mathrm{B} \alpha$, and (D) p-p65 (mean $\pm \mathrm{SD},{ }^{*} p<0.05$ compared to the LPS-treated group). C: control group treated with 0.5\% DMSO. 1,5-dimethyl citrate (1).

\subsection{Compound 1 downregulated iNOS and COX-2 expression in LPS-stimulated RAW 264.7 mouse macrophages}

The induction of iNOS and COX-2 during inflammation directly contributes to NO synthesis [31]. Therefore, we proceeded to verify whether compound 1 (1,5-dimethyl citrate) affects LPS-stimulated iNOS and COX-2. As shown in Figure 5, exposure to $1 \mu \mathrm{g} / \mathrm{mL}$ LPS significantly upregulated iNOS and COX-2; however, pretreatment with 50 and $100 \mu \mathrm{M}$ of compound 1 could reverse the changes induced by LPS. Compound 1 could therefore inhibit the increase in iNOS and COX-2 induced via the NF- $\kappa B$ signaling pathway. On the basis of these results, the suppression in NO production of compound $\mathbf{1}$ might be associated with downregulating the expression of iNOS and COX-2 by LPS stimulation in RAW 264.7 macrophages.

(A)

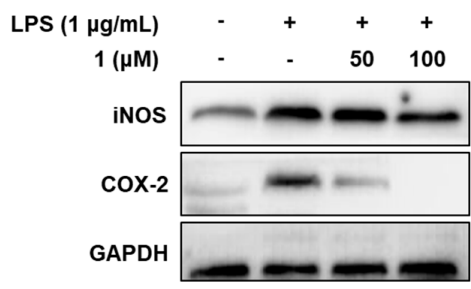

(B)

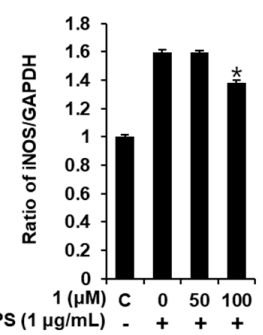

(C)

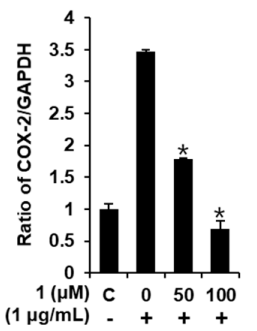

Figure 5. Effects of compound 1 on the LPS-induced expression of iNOS and COX-2 in RAW 264.7 mouse macrophages. (A) Representative Western blots of iNOS, COX-2, and GAPDH protein expression. Quantitative graph of (B) iNOS and (C) COX-2 (mean \pm SD, ${ }^{*} p<0.05$ compared to the LPS-treated group). C: control group treated with 0.5\% DMSO. 1,5-dimethyl citrate (1). 
3.6. Compound 1 downregulated IL-6 and TNF- $\alpha$ production in LPS-stimulated RAW 264.7 mouse macrophages

Elevated expression levels of iNOS and COX-2 followed by an increase in the production of NO have been found to be evoked by TNF- $\alpha$ and IL-6 in RAW 264.7 macrophages [32]. Lastly, we proceeded to examine whether compound 1 affected the production of pro-inflammatory cytokines, including IL-6 and TNF- $\alpha$, in LPS-stimulated RAW 264.7 mouse macrophages. As shown in Figure 6, treating RAW 264.7 macrophages with LPS markedly increased IL- 6 and TNF- $\alpha$ production, which were ameliorated by 50 and $100 \mu \mathrm{M}$ of compound $\mathbf{1}$ in a concentration-dependent manner. These results suggest that compound 1 inhibits IL- 6 and TNF- $\alpha$ production, ultimately leading to a reduction in inflammatory response. Altogether, we found that the inhibition of NO production in LPS-activated RAW 264.7 macrophages by compound 1 was mediated by the inhibition of $I K K \alpha / \beta, I-\kappa B \alpha, N F-\kappa B$ p65, iNOS, and COX-2, as well as the activities of IL-6 and TNF- $\alpha$ (Figure 7).

(A)

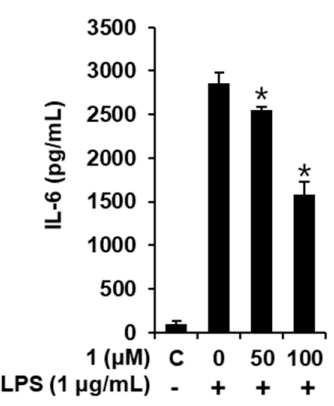

(B)

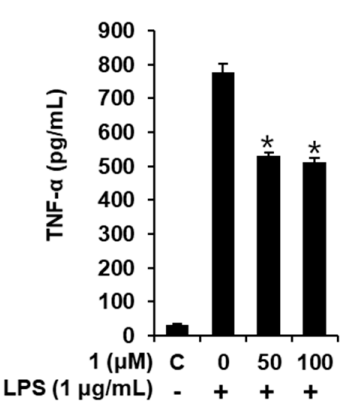

Figure 6. Effects of compound 1 on the LPS-induced cytokine release in RAW 264.7 mouse macrophages. (A) Effect of compound 1 on LPS-induced IL-6 production. (B) Effects of compound 1 on LPS-induced TNF- $\alpha$ production (mean $\pm \mathrm{SD}, * p<0.05$ compared to the LPS-treated group). C: control group treated with $0.5 \%$ DMSO. 1,5-dimethyl citrate (1).

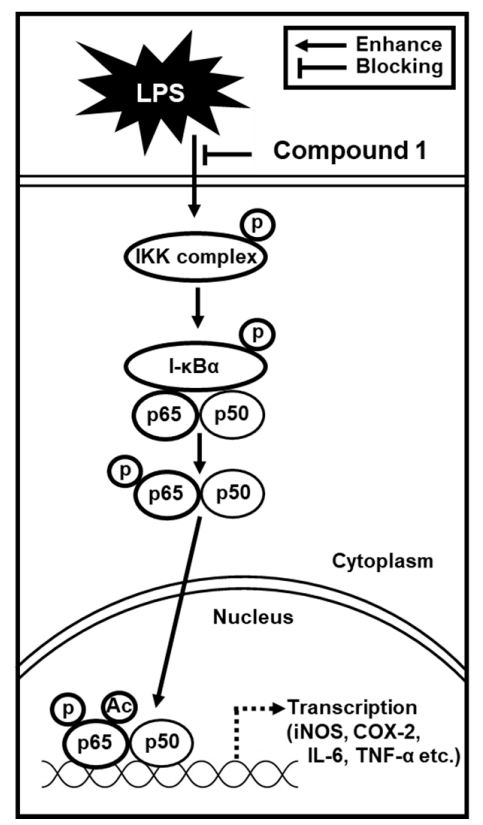

Figure 7. Schematic model showing the inhibitory effects of compound $\mathbf{1}$ on lipopolysaccharide-induced inflammatory response in RAW 264.7 mouse macrophages through the inhibition of IKK $\alpha / \beta, \mathrm{I}-\kappa \mathrm{B} \alpha$, NF- $\mathrm{kB}$ p65, inducible nitric oxide synthase (iNOS), and cyclooxygenase-2 (COX-2), and the activities of IL-6 and TNF- $\alpha$. 1,5-dimethyl citrate (1). 
In previous studies, flavonoids as main components of sea buckthorn (H. rhamnoides) including isorhamnetin, quercetin, and kaempferol, inhibited LPS-induced NO production in RAW 264.7 cells through the inhibition of expressions of iNOS, COX-2, proteins in the mitogen-activated protein kinase (MAPK) pathway (c-Jun N-terminal kinase and $\mathrm{p} 38$ ), I- $\mathrm{kB} \alpha, \mathrm{NF}-\mathrm{kB}$ p65, and production of pro-inflammatory cytokines (IL-6, IL-1 $\beta$, and TNF- $\alpha$ ) [33]. The mechanism of action of flavonoids from sea buckthorn was similar to that of 1,5-dimethyl citrate (1) that we identified in this study. However, it has not been known exactly which flavonoid of sea buckthorn exhibits the anti-inflammatory effect. To the best of our knowledge, this study is the first to evaluate the anti-inflammatory activity of citric acid derivative isolated from sea buckthorn on LPS-induced inflammatory response in RAW 264.7 mouse macrophages. The active compound, 1,5-dimethyl citrate (1), was a simple derivative of citric acid present in many plants and fruits [34-37]. An anti-inflammatory effect of citric acid derivatives has been reported in previous studies [34-37]. In LPS-treated mice, increased TNF- $\alpha$ in mice brain tissue and iNOS expression in the cytoplasm of hepatocytes were decreased after treatment of citric acid [34]. In a recent study, wheat germ extract with citric acid was reported to inhibit secretion of the pro-inflammatory cytokines, TNF- $\alpha$, IL-6, and IL-12 and the synthesis of COX-2, as well as phosphorylation of NF-KB p65 and p38 kinase in LPS-activated macrophages [35]. In addition, calcium citrate showed antioxidant enzyme activities, and inhibited NO production by suppression of the expression of pro-inflammatory mediators (NF- $\mathrm{KB}$, iNOS, and COX-2) and cytokines (TNF- $\alpha$, IL-1 $\beta$, and IL-6) in LPS-stimulated RAW 264.7 macrophages [36]. Another recent study reported that citric acid displayed anti-inflammatory function via the toll-like receptor (TLR)-mediated activation of NF- $\kappa B$ and interferon regulatory factor- 3 signaling pathways, and that citric acid contributed to the maintenance of tight junction proteins via the TLR-mediated p38 and c-Jun N-terminal kinase pathways [37]. Interestingly, a new oligosaccharide citric acid derivative isolated from Vigna angularis (ohwi et ohashi. var. Dainagon) seeds displayed promising anti-influenza A virus activity [38]. Additionally, a recent paper reported that citric acid showed protective effect against hepatic ischemia reperfusion injury in Sprague-Dawley rats, which suggests significant therapeutic potential of citric acid in ischemic liver injury [39]. Although future in vivo studies should support the anti-inflammatory effects of 1,5-dimethyl citrate (1), identified as an active compound in this study, our findings suggest a potential application of 1,5-dimethyl citrate (1) for the treatment of inflammatory diseases.

\section{Conclusions}

Herein, we provided experimental evidence of the potential role of 1,5-dimethyl citrate from $H$. rhamnoides fruits in the management and treatment of inflammatory diseases. By performing a phytochemical analysis of the extracts of $H$. rhamnoides fruits using repeated column chromatography, HPLC, and LC/MS, we isolated and identified six compounds (1-6), namely, a citric acid derivative (1), a phenolic (2), two flavonoids ( 3 and 4 ), and two megastigmane compounds (5 and 6). Among them, 1,5-dimethyl citrate (1) was demonstrated as effectively preventing LPS-induced NO production and markedly inhibited the expression of IKK $\alpha / \beta, \mathrm{I}-\kappa \mathrm{B} \alpha, \mathrm{NF}-\mathrm{kB}$ p 65 , iNOS, and COX-2, and the activities of IL- 6 and TNF- $\alpha$. On the basis of our findings, we have provided experimental evidence that the 1,5-dimethyl citrate (1) obtained from $H$. rhamnoides fruits could function as an effective agent for the treatment of inflammatory diseases.

Supplementary Materials: The following are available online at http://www.mdpi.com/2304-8158/9/3/269/s1: Figure S1: ECD data of compounds 5 (A) and 6 (B), Figure S2: LC/MS-based analysis (detection wavelength was set as $210 \mathrm{~nm}$ ) of the ethyl acetate-soluble fraction as well as the chemical structures of compounds 1-4, Figure S3: LC/MS-based analysis (detection wavelength was set as $210 \mathrm{~nm}$ ) of the $\mathrm{CH} 2 \mathrm{Cl2}$-soluble fraction as well as the chemical structures of compounds 5-6, Figure S4: LC/MS analysis for purity of compounds 1-6.

Author Contributions: Conceptualization, K.S.K., N.Y., and K.H.K.; formal analysis, S.C.B., D.L., M.S.J., K.H.L., Y.H.L., and K.H.K.; investigation, S.C.B. and D.L.; writing-original draft preparation, S.C.B., D.L., K.S.K., and K.H.K.; writing一review and editing, S.C.B., N.Y., and K.H.K.; project administration, K.S.K., N.Y., and K.H.K.; fund acquisition, K.H.K. All authors have read and agreed to the published version of the manuscript. 
Funding: This work was supported by a grant from the National Research Foundation of Korea (NRF), funded by the Korean government (MSIT) (2018R1A2B2006879 and 2019R1A5A2027340).

Acknowledgments: We would like to thank Editage (www.editage.co.kr) for English language editing.

Conflicts of Interest: The authors have no conflict of interest to declare.

\section{References}

1. Hussain, I.; Khan, L.; Gul, A.; Ahmed, N.; Saleem, M. Comparative study of vitamin C contents in fruits and medicinal plants. J. Chem. Soc. Pak. 2008, 30, 406-409.

2. Xu, X.; Gao, Y.; Liu, G.; Wang, Q.; Zhao, J. Optimization of supercritical carbon dioxide extraction of sea buckthorn (Hippophae rhamnoides L.) oil using response surface methodology. LWT Food Sci. Technol. 2008, 41, 1223-1231. [CrossRef]

3. Yang, B.R.; Kalimo, K.O.; Tahvonen, R.L.; Matilla, L.M.; Katajisto, J.K.; Kallio, H.P. Effect of dietary supplementation with sea buckthorn (Hippophae rhamnoides) seed and pulp oils on the fatty acid composition of skin glycerophospholipids patients with atopic dermatitis. J. Nutr. Biochem. 2000, 11, 338-340. [CrossRef]

4. Zhou, W.; Yuan, Z.; Li, G.; Ouyang, J.; Suo, Y.; Wang, H. Isolation and structure determination of a mew flavone glycoside from seed residues of seabuckthorn (Hippophae rhamnoides L.). Nat. Prod. Res. 2018, 32, 892-897. [CrossRef]

5. Skalski, B.; Kontek, B.; Rolnik, A.; Olas, B.; Stochmal, A.; Żuchowski, J. Anti-platelet properties of phenolic extracts from the leaves and twigs of Elaeagnus rhamnoides (L.) A. Nelson. Molecules 2019, 24, 3620. [CrossRef]

6. Różalska, B.; Sadowska, B.; Żuchowski, J.; Więckowska-Szakiel, M.; Budzyńska, A.; Wójcik, U.; Stochmal, A. Phenolic and nonpolar fractions of Elaeagnus rhamnoides (L.) A. Nelson, extracts as virulence modulators-in vitro study on bacteria, fungi, and epithelial cells. Molecules 2018, 23, 1498. [CrossRef]

7. Rösch, D.; Mügge, C.; Fogliano, V.; Kroh, L.W. Antioxidatn oligomeric proanthocyanidins from sea buckthorn (Hippophaë rhamnoides) pomace. J. Agric. Food Chem. 2004, 52, 6712-6718. [CrossRef]

8. OuYang, J.; Zhou, W.N.; Li, G.; Wang, X.Y.; Ding, C.X.; Suo, Y.R.; Wang, H.L. Three New Alkaloids from Hippophae rhamnoides Linn. subsp. sinensis Rousi. Helv. Chim. Acta. 2015, 98, 1287-1291. [CrossRef]

9. Gutzeit, D.; Wray, V.; Winterhalter, P.; Jerz, G. Preparative isolation and purification of flavonoids and protocatechuic acid from sea buckthorn juice concentrate (Hippophä rhamnoides L. ssp. rhamnoides) by high-speed counter-current chromatography. Chromatogrphia 2007, 65, 1-7.

10. Chen, C.; Gao, W.; Cheng, L.; Shao, Y.; Kong, D.Y. Four new triterpenoid glycosides from the seed residue of Hippophae rhamnoides subsp sinensis. J. Asian Nat. Prod. Res. 2014, 16, 231-239. [CrossRef]

11. Zhang, G.; Liu, Y.; Liu, P. Active components from sea buckthorn (Hippophae rhamnoides L.) regulate hepatic stellate cell activation and liver fibrogenesis. J. Agric. Food Chem. 2018, 66, 12257-12264. [CrossRef]

12. Lau, T.A.; Bray, W.M.; Lokey, R.S. Macrophage Cytological Profiling and Anti-Inflammatory Drug Discovery. Assay Drug Dev. Techn. 2019, 17, 14-16. [CrossRef]

13. Azab, A.; Nassar, A.; Azab, A.N. Anti-inflammatory activity of natural products. Molecules 2016, $21,1321$. [CrossRef]

14. Chen, L.; Deng, H.; Cui, H.; Fang, J.; Zuo, Z.; Deng, J.; Li, Y.; Wang, X.; Zhao, L. Inflammatory responses and inflammation-associated diseases in organs. Oncotarget 2018, 9, 7204. [CrossRef]

15. Hirayama, D.; Iida, T.; Nakase, H. The phagocytic function of macrophage-enforcing innate immunity and tissue homeostasis. Int. J. Mol. Sci. 2018, 19, 92. [CrossRef]

16. Cavaillon, J.M. Exotoxins and endotoxins: Inducers of inflammatory cytokines. Toxicon 2018, 149, 45-53. [CrossRef]

17. Murphy, M.P. Nitric oxide and cell death. Biochim. Biophys. Acta 1999, 1411, 401-414. [CrossRef]

18. Liu, T.; Zhang, L.; Joo, D.; Sun, S.-C. NF-кB signaling in inflammation. Signal. Transduc. Target. Ther. 2017, 2, 17023. [CrossRef] [PubMed]

19. So, H.M.; Eom, H.J.; Lee, D.; Kim, S.; Kang, K.S.; Lee, I.K.; Baek, K.-H.; Park, J.Y.; Kim, K.H. Bioactivity evaluations of betulin identified from the bark of Betula platyphylla var. japonica for cancer therapy. Arch. Pharm. Res. 2018, 41, 815-822. [PubMed]

20. Yu, J.S.; Roh, H.-S.; Baek, K.-H.; Lee, S.; Kim, S.; So, H.M.; Moon, E.; Pang, C.; Jang, T.S.; Kim, K.H. Bioactivity-guided isolation of ginsenosides from Korean Red Ginseng with cytotoxic activity against human lung adenocarcinoma cells. J. Ginseng Res. 2018, 42, 562-570. [CrossRef] [PubMed] 
21. Baek, S.C.; Choi, E.; Eom, H.J.; Jo, M.S.; Kim, S.; So, H.M.; Kim, S.H.; Kang, K.S.; Kim, K.H. LC/MS-based analysis of bioactive compounds from the bark of Betula platyphylla var. japonica and their effects on regulation of adipocyte and osteoblast differentiation. Nat. Prod. Sci. 2018, 24, 235-240.

22. Yu, J.S.; Lee, D.; Lee, S.R.; Lee, J.W.; Choi, C.-I.; Jang, T.S.; Kang, K.S.; Kim, K.H. Chemical characterization of cytotoxic indole acetic acid derivative from Mulberry fruit (Morus alba L.) against human cervical cancer. Bioorg. Chem. 2018, 76, 28-36. [CrossRef]

23. Choi, J.H.; Lee, D.U. Practical synthesis of novel citryl glycoside, the component of the fhizomes of Gastrodia elata. Bull. Korean Chem. Soc. 2008, 29, 2051-2053.

24. Zhang, Y.H.; Yu, J.Q. Pd(II)-catalyzed hydroxylayion of arenes with 1 atm of O2 or air. J. Am. Chem. Soc. 2009, 131, 14654-14655. [CrossRef] [PubMed]

25. Moco, S.; Tseng, L.H.; Spraul, M.; Chen, Z.; Vervoort, J. Building-up a comprehensive database of flavonoids based on nuclear magnetic resonance data. Chromatographia 2006, 64, 503-508. [CrossRef]

26. Kai, H.; Baba, M.; Okuyama, T. Two new megastigmanes from the leaves of Cucumis sativus. Chem. Pharm. Bull. 2007, 55, 133-136. [CrossRef]

27. Yamano, Y.; Ito, M. Synthesis of optically active vomifoliol and roseoside stereoisomers. Chem. Pharm. Bull. 2005, 53, 541-546. [CrossRef]

28. McKenna, S.; Wright, C.J. Inhibiting I $\kappa \beta \beta-N F \kappa B$ signaling attenuates the expression of select pro-inflammatory genes. J. Cell Sci. 2015, 128, 2143-2155. [CrossRef]

29. Dorrington, M.G.; Fraser, I.D. NF-kB signaling in macrophages: Dynamics, crosstalk, and signal integration. Front. Immunol. 2019, 10, 705. [CrossRef]

30. Hayden, M.S.; Ghosh, S. NF-кB in immunobiology. Cell Res. 2011, 21, 223-244. [CrossRef]

31. Murakami, A.; Ohigashi, H. Targeting NOX, INOS and COX-2 in inflammatory cells: Chemoprevention using food phytochemicals. Int. J. Cancer 2007, 121, 2357-2363. [CrossRef] [PubMed]

32. Yahfoufi, N.; Alsadi, N.; Jambi, M.; Matar, C. The Immunomodulatory and Anti-Inflammatory Role of Polyphenols. Nutrients 2018, 10, 1618. [CrossRef] [PubMed]

33. Jiang, F.; Guan, H.; Liu, D.; Wu, X.; Fan, M.; Han, J. Flavonoids from sea buckthorn inhibit the lipopolysaccharide-induced inflammatory response in RAW264.7 macrophages through the MAPK and NF-kB pathways. Food Funct. 2017, 8, 1313-1322. [CrossRef] [PubMed]

34. Abdel-Salam, O.M.E.; Youness, E.R.; Mohammed, N.A.; Morsy, S.M.Y.; Omara, E.A.; Sleem, A.A. Citric Acid Effects on Brain and Liver Oxidative Stress in Lipopolysaccharide-Treated Mice. J. Med. Food. 2014, 17, 588-598. [CrossRef] [PubMed]

35. Jeong, H.Y.; Choi, Y.S.; Lee, J.K.; Lee, B.J.; Kim, W.K.; Kang, H. Anti-Inflammatory Activity of Citric Acid-Treated Wheat Germ Extract in Lipopolysaccharide-Stimulated Macrophages. Nutrients 2017, 9, 730. [CrossRef]

36. Choi, E.Y.; Kim, H.J.; Han, J.S. Anti-inflammatory effects of calcium citrate in RAW 264.7cells via suppression of NF-кB activation. Environ. Toxicol. Pharmacol. 2015, 39, 27-34. [CrossRef]

37. Zhao, S.; Chen, Z.; Zheng, J.; Dai, J.; Ou, W.; Xu, W.; Ai, Q.; Zhang, W.; Niu, J.; Mai, K.; et al. Citric acid mitigates soybean meal induced inflammatory response and tight junction disruption by altering TLR signal transduction in the intestine of turbot, Scophthalmus maximus L. Fish Shellfish Immunol. 2019, 92, 181-187. [CrossRef]

38. Mohammed, M.M.D.; Kobayashi, N. Anti-Influenza a virus of a new oligosaccharide citric acid derivative isolated from Vigna angularis (ohwi et ohashi. var. Dainagon) seeds. J. Carbohyd. Chem. 2019, 38, 234-245. [CrossRef]

39. Kim, S.K.; Kang, S.W.; Jin, S.A.; Ban, J.Y.; Hong, S.J.; Park, M.S. Protective effect of citric acid against hepatic ischemia reperfusion injury in Sprague-Dawley rats. Transplant. Proc. 2019, 51, 2823-2827. [CrossRef]

(C) 2020 by the authors. Licensee MDPI, Basel, Switzerland. This article is an open access article distributed under the terms and conditions of the Creative Commons Attribution (CC BY) license (http://creativecommons.org/licenses/by/4.0/). 